\title{
A Vision and Roadmap for Increasing User Autonomy in Flight Operations in the National Airspace
}

\author{
Capt. William B. Cotton ${ }^{1}$, Capt. Robert Hilb ${ }^{2}$ \\ National Institute of Aerospace, Hampton, VA 23666 \\ Stefan $\mathrm{Koczo}^{3}$ \\ Cedar Rapids, IA 52498 \\ David Wing ${ }^{4}$ \\ NASA Langley Research Center, Hampton, VA 23681, USA
}

\begin{abstract}
The purpose of Air Transportation is to move people and cargo safely, efficiently and swiftly to their destinations. The companies and individuals who use aircraft for this purpose, the airspace users, desire to operate their aircraft according to a dynamically optimized business trajectory for their specific mission and operational business model. In current operations, the dynamic optimization of business trajectories is limited by constraints built into operations in the National Airspace System (NAS) for reasons of safety and operational needs of the air navigation service providers. NASA has been developing and testing means to overcome many of these constraints and permit operations to be conducted closer to the airspace user's changing business trajectory as conditions unfold before and during the flight. A roadmap of logical steps progressing toward increased user autonomy is proposed, beginning with NASA's Traffic Aware Strategic Aircrew Requests (TASAR) concept that enables flight crews to make informed, deconflicted flight-optimization requests to air traffic control. These steps include the use of data communications for route change requests and approvals, integration with time-based arrival flow management processes under development by the Federal Aviation Administration (FAA), increased user authority for defining and modifying downstream, strategic portions of the trajectory, and ultimately application of self-separation. This progression takes advantage of existing FAA NextGen programs and RTCA standards development, and it is designed to minimize the number of hardware upgrades required of airspace users to take advantage of these advanced capabilities to achieve dynamically optimized business trajectories in NAS operations. The roadmap is designed to provide operational benefits to first adopters so that investment decisions do not depend upon a large segment of the user community becoming equipped before benefits can be realized. The issues of equipment certification and operational approval of new procedures are addressed in a way that minimizes their impact on the transition by deferring a change in the assignment of separation responsibility until a large body of operational data is available to support the safety case for this change in the last roadmap step. This paper will relate the roadmap steps to ongoing activities to clarify the economics-based transition to these technologies for operational use.
\end{abstract}

$\mathrm{U}$ sers of the National Airspace System (NAS) - airlines, general aviation, unmanned vehicles, and military - all have specific objectives for their respective operations that vary widely in purpose, but each benefits from the degree of operational autonomy they are permitted in the performance of their individual missions. In order to provide safety in these flight operations, extensive and restrictive rules and procedures have emerged over many decades that limit or constrain the operations to the degree required by human and other limitations in the systems that have safety responsibility. Thus the air traffic control system, the organization of the airspace, and the rules and procedures governing all flight operations have been created with the primary objective of providing the safety function and secondarily the function of expediting traffic flow.

An unfortunate byproduct of the current system for ensuring safety in flight operations is an abundance of constraints that have evolved and become necessary over time to compensate for the increasing numbers of flights

\footnotetext{
${ }^{1}$ Aviation Subject Matter Expert, Cotton Aviation Enterprises, wmcotton@ gmail.com

2 Aviation Subject Matter Expert, Bob Hilb, LLC, bhilb@cableone.net

${ }^{3}$ Principal Systems Engineer - Retired, Rockwell Collins Advanced Technology Center, swkoczo@gmail.com

${ }^{4}$ ATM Research Engineer, Crew Systems \& Aviation Operations, Mail Stop 152, david.wing@ nasa.gov, AIAA member
} 
needed to accommodate the demand for air transportation. This is made worse by the inability to adequately forecast and respond to uncertainties associated with operations in the NAS, including the impact of adverse weather conditions and other disruptive factors in the airspace. The frequent results are significant delays, inefficiency, and temporary denial of access to much of the NAS for many existing and potential users. This occurs while the airspace itself, the wide open skies, the most abundant national resource there is, goes largely unused. NASA has for decades sought means for overcoming some of these constraints in a way that will ensure safety through alternate means while enabling more dynamic optimization by airspace users of each of their individual missions.

NASA's Aeronautics Research Mission Directorate is focusing on operational autonomy in the Next Generation Air Transportation System (NextGen) and beyond. The vision for achieving this autonomy makes use of satellite positioning, data communications, access to NAS operational and environmental data, and distributed processing to enable the highest degree of operational autonomy while continuing to improve safety, but doing it in a pragmatic manner that can be afforded by both airspace users and air navigation service providers. Entirely new operational concepts for both manned and unmanned aircraft systems can be enabled by this autonomy to unleash significant future operational capabilities.

Yet it is not possible, or even desirable, to proceed in a single leap from current operations to fully autonomous operations in the NAS. Instead, it is instructive to examine varying degrees of autonomy in different operations and a series of well-planned steps of increasing operational capabilities that build on each other to achieve the future vision in effective operations. Visual Flight Rules (VFR) provides one example of existing flight operations that enjoy a high degree of autonomy. With the responsibility to see and avoid other aircraft, VFR flights are permitted to operate in Class E and G airspace with no communications with either Air Traffic Control (ATC) or the other aircraft sharing this airspace. Employing sensors and automation, enabled by data communications and information management to perform the safety function with very high integrity and reliability, could enable such autonomy in all classes of airspace. In another example, small Unmanned Aircraft System (UAS) operations may be conducted at low altitude as long as the operator maintains visual contact with the aircraft and controls it so as not to create a hazard to other aircraft, persons or property. Radio-controlled model aircraft have been flown this way for many decades. Similar use of sensors and automation could safely expand these operations beyond line of sight and remove the need for human pilot control of the vehicle. Both of these examples show the potential for stepwise implementation of broadly-based airborne vehicle autonomy using automation.

Locating the surveillance and automation elements of control in the aircraft to satisfy the safety requirements of the separation function is still considered to be a major step into the unknown. This is true, even though one intuitively knows that computer systems can function far more reliably doing complex repetitive tasks than humans, and they do so in most other aspects of our lives, routinely. An example of this is automatic braking in many new car models to assist in preventing collisions. Building confidence in separation automation will take time as operational experience is gained and the limitations and design flaws in new equipment are identified and corrected. By trying out the conflict detection and resolution algorithms in limited situations, this experience can be accumulated and confidence will grow. It is also true that the presence of increasingly autonomous operations in the airspace currently being solely managed by human air traffic controllers will constitute a cultural shift for those controllers that will take some time getting used to, even though they will continue to fill their current role for much of the traffic for the foreseeable future. Building up these operations through a series of well-planned, deliberate steps should build confidence in such systems and ease the objections and angst of the cultural change they represent. Finally, the assumption of responsibility for separation safety by automated systems creates issues of safety assessment, certification, and operational approval that have not previously been dealt with. This paper examines these issues in depth and proposes a roadmap of airborne system and procedural development activities leading to autonomous operations in a safe and broadly acceptable way. This roadmap is referred to as Airborne Trajectory Management (ABTM) in this paper to denote the significant role and benefits provided by an increasingly autonomous series of aircraft-based capabilities and applications that are expected to overcome the constraints and limitations of the NAS. The roadmap is designed, as much as possible, to provide sufficient monetary benefit to the early adopters - those first to make the needed investments in avionics upgrades and flight crew training for software applications and procedures that provide these new capabilities.

In presenting the logical, functional steps in the ABTM Roadmap to this vision for increased operational autonomy, the paper details the requirements placed on the airspace users, the air navigation service providers, the standards organizations, and the rule makers for each step in the transition. The roadmap begins with the "Traffic Aware Strategic Aircrew Requests" (TASAR) $)^{1,2}$ application, the first basic step in dynamic ABTM, and ends at the "Autonomous Flight Rules" (AFR) 3,4 $^{3,4}$ concept that maximizes flexibility and access in the NAS for the airspace user, 
while preserving and ensuring flight safety for all users, whether or not they are participating in the applications of increased autonomy.

\section{Attributes of Roadmap Design}

The design philosophy used in the creation of the roadmap was formulated during an analysis of the benefits and challenges associated with the many proposed applications and concepts of operation using airborne surveillance. This analysis considered currently approved applications, applications under development, and advanced concepts that were the result of a function-allocation analysis of the roles of air and ground automation and personnel related to trajectory management, strategic conflict avoidance, and tactical separation assurance that must be accomplished with a high level of integrity to assure operational safety. Each of the surveillance and trajectory management concepts was assessed for its ability to achieve the goals of increased operating efficiency and flexibility through increasing user autonomy.

The design philosophy also included seeking "first adopter benefits," thereby not requiring many aircraft to be equipped before monetary benefits needed for return-on-investment could be realized by those who did equip. The philosophy also leverages existing FAA programs for NextGen, like the Data Communications (Data Comm) program and the Automatic Dependent Surveillance-Broadcast (ADS-B) (OUT/IN) program with application categories like Advanced Interval Management (A-IM). While not dependent on these to attain benefit, each integration step provides additional benefit beyond the previous step. The design philosophy also delays paradigm shifts in the control of air traffic that could create barriers to transition. It is only after extensive experience has been gained through operations in the earlier steps that rules and procedures would be changed to fully exploit new capabilities. Even if the final vision is never realized, each step in the roadmap on its own represents a worthwhile improvement in its own right.

\section{Approach}

All existing and proposed applications of airborne surveillance and trajectory management were assembled and analyzed for their ability to contribute to the advancement of autonomy in flight operations. These applications came from existing and proposed FAA development programs, from the final report of the ADS-B IN Aviation Rulemaking Committee, ${ }^{5}$ from NASA's research on advanced air traffic services, Air Traffic Management (ATM), and separation technology concepts, ${ }^{6,7,8}$ and subsequent determination of "function allocation" alternatives which led to identification of logical combinations and extensions of these concepts. Each concept and application was vetted for its positive attributes - efficiency, flexibility, and return-on-investment, and its potential negative aspects of cost and difficulty in achieving implementation. Each application was also examined for its current level of maturity and for the status and timing of standards development that would need to be developed to achieve future equipment certification and operational approval.

A matrix of applications and concepts describing their attributes, benefits, and implementation difficulties was created and used to evaluate and down-select candidates by overall value, resulting in an orderly progression of capabilities for improving operational autonomy, i.e., the ABTM Roadmap described in this paper. From this effort, a progression of five steps emerged to form a viable and practical roadmap for increasing operational autonomy through the process of Airborne Trajectory Management. The steps are titled and summarized as follows:

1. Basic TASAR, using automation to compute optimized lateral and vertical route changes for voice request

2. Digital TASAR, adding data communications

3. 4D (Four Dimensional) TASAR, adding the speed and time dimension

4. Strategic ABTM, adding authority to update the strategic trajectory (downstream sectors)

5. Full ABTM, adding separation responsibility and authority to make tactical trajectory changes (current sector)

\section{Roadmap for Increasing Operational Autonomy}

This section describes the ABTM Roadmap steps in order of increasing capability and autonomy. Each step adds to the ability of an operator to dynamically optimize its flights within the overall NAS environment through increasing use of ABTM. Individual airspace users may choose to implement the full regimen of steps over time or may stop at an intermediate level of capability, depending on their type of operations and their unique cost and benefit factors. 


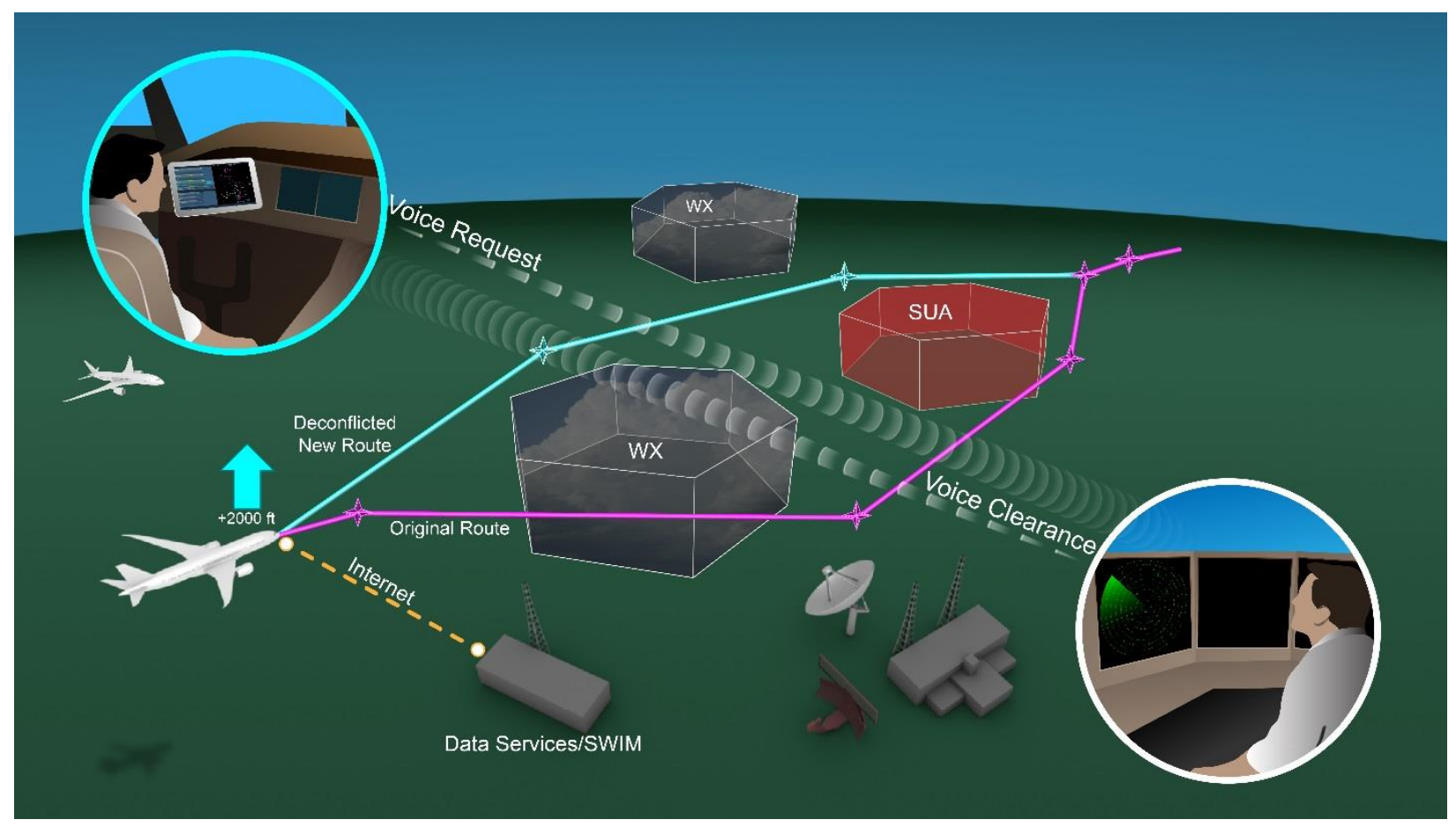

Figure 1. ABTM Roadmap Step 1-Basic TASAR.

ABTM 1. Traffic Aware Strategic Aircrew Requests (Basic TASAR): Depicted in Fig. 1, Basic TASAR is an existing Electronic Flight Bag (EFB)-based, trajectory optimization program developed by NASA that uses current, own-ship aircraft performance data, external traffic information, and weather and airspace constraint data to produce trajectory-change recommendations that best meet the operator's objectives for fuel and/or time savings that are more approvable by ATC, given the inclusion of traffic, weather, and airspace constraint data. The system outputs lateral and vertical path changes, called "solutions," that are filtered to prevent proposing traffic-conflicted change requests (based on traffic awareness using airborne surveillance), to avoid weather hazards, and to avoid airspace constraints such as Special Use Airspace (SUA). Similarly, fixed ATC constraints and static ATC-preferred routings are accommodated. The optimization tool, a NASA-developed prototype which is called the Traffic Aware Planner (TAP), ${ }^{8}$ uses aircraft surveillance information, e.g., from ADS-B OUT, to reject solutions that are conflicted by other aircraft. The solutions are presented to the pilot in a graphical form on the EFB, overlaid on the active route for comparison. The fuel and time savings outcomes are also displayed for the pilots' evaluation and, if desired, request to ATC for approval. The suggested trajectory changes from Basic TASAR are also kept simple enough that voice requests by pilots and approvals by controllers are operationally acceptable using current-day user request procedures. As this procedure requires no changes to ATC procedures, systems, or responsibilities, it keeps the barrier to approval of the concept very low, while allowing first adopters to immediately gain modest benefits for each equipped aircraft.

Figure 2 shows a block diagram of an EFB implementation of Basic TASAR, the current architecture pursued to date by the Basic TASAR development team. Figure 3 shows an alternative integration of the TAP software with certified avionics that more readily accommodates the later steps in the Roadmap. Basic TASAR has been extensively and successfully flight tested and is about to undergo operational evaluation by NASA and their partner airlines, Alaska Airlines and Virgin America, in revenue operations. ${ }^{9}$ A preliminary safety assessment of Basic TASAR determined its likely Failure Effects Classification (FEC) to be "No Effect" and no higher than "Minor" due to potential pilot workload, an assessment to which FAA policy officials briefed on Basic TASAR concurred. ${ }^{10}$ An extension of the preliminary safety assessment to the applications in this roadmap determined that the next two steps, Digital TASAR and 4D TASAR, would have the same FEC as Basic TASAR. ${ }^{11}$ 


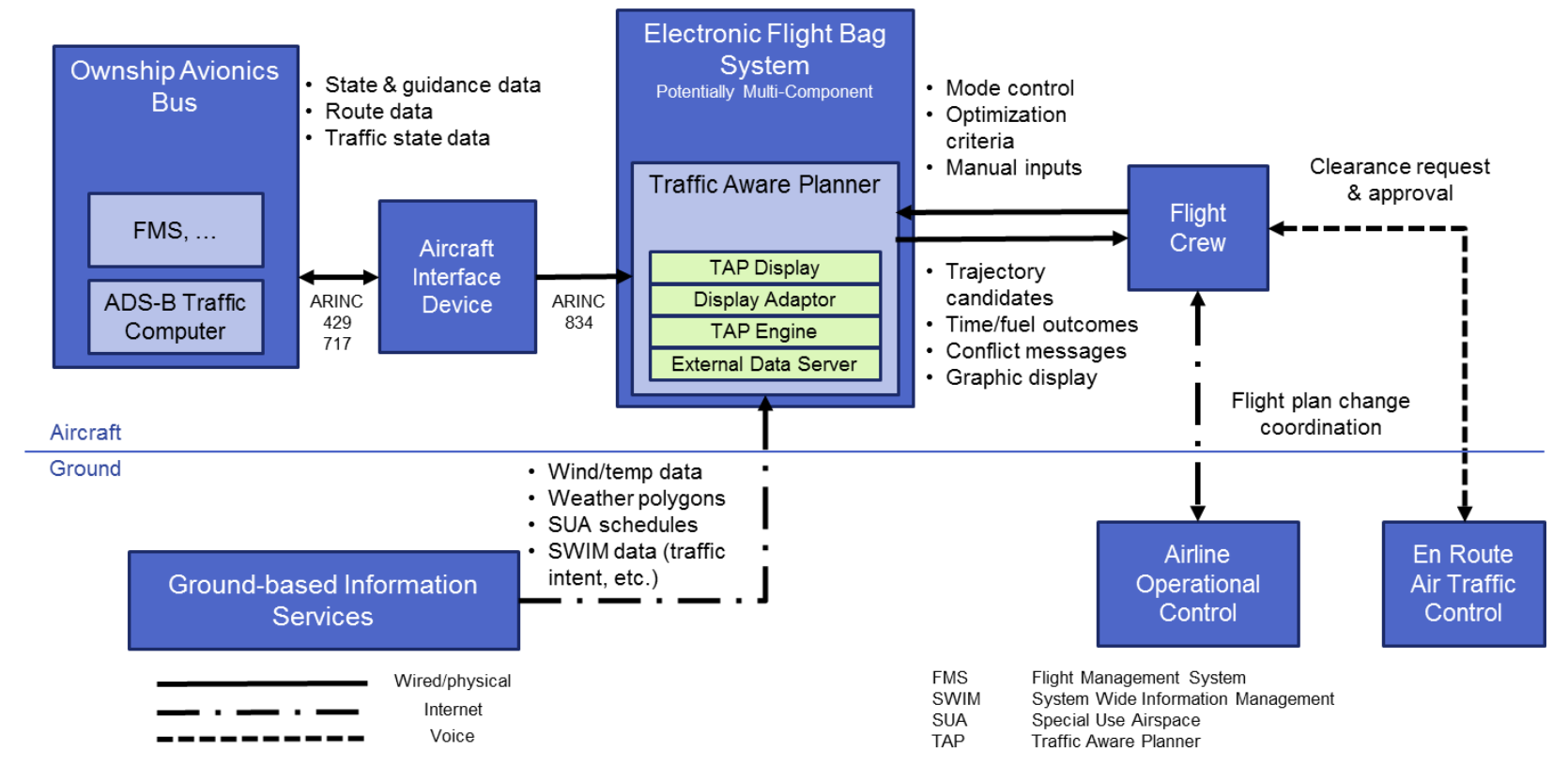

Figure 2. An EFB-based avionics architecture suitable for Basic TASAR.



Figure 3. ABTM avionics architecture based on integrated avionics.

ABTM 2. Digital TASAR: Depicted in Fig. 4, Digital TASAR is the second step on the ABTM Roadmap, an upgrade of Basic TASAR using data links for requests and re-clearance to permit the system to generate longer and more complex trajectory change opportunities to optimize the flight-time and fuel-burn objectives of the flight. In this step, the optimization flight regime is extended from the initial regime of essentially en route cruise to now include the departure and arrival phases. On a great many flights, the cruise segment is less than one third of the total flight time, making the climb and decent portions prime targets for optimization, not only taking advantage of more up-todate winds, but vertical wind gradients as well. In this application, dynamic trajectory optimization can include longer and more complex trajectory / route graphical descriptions because they are requested and approved using data communications between the aircraft and the ATC system. Neither pilots nor controllers need to speak or manually enter the route description in this data exchange. The solutions are more valuable and more robust, and they reduce the workload on both pilots and controllers in the request/approval process. Digital TASAR makes greater use of

American Institute of Aeronautics and Astronautics 


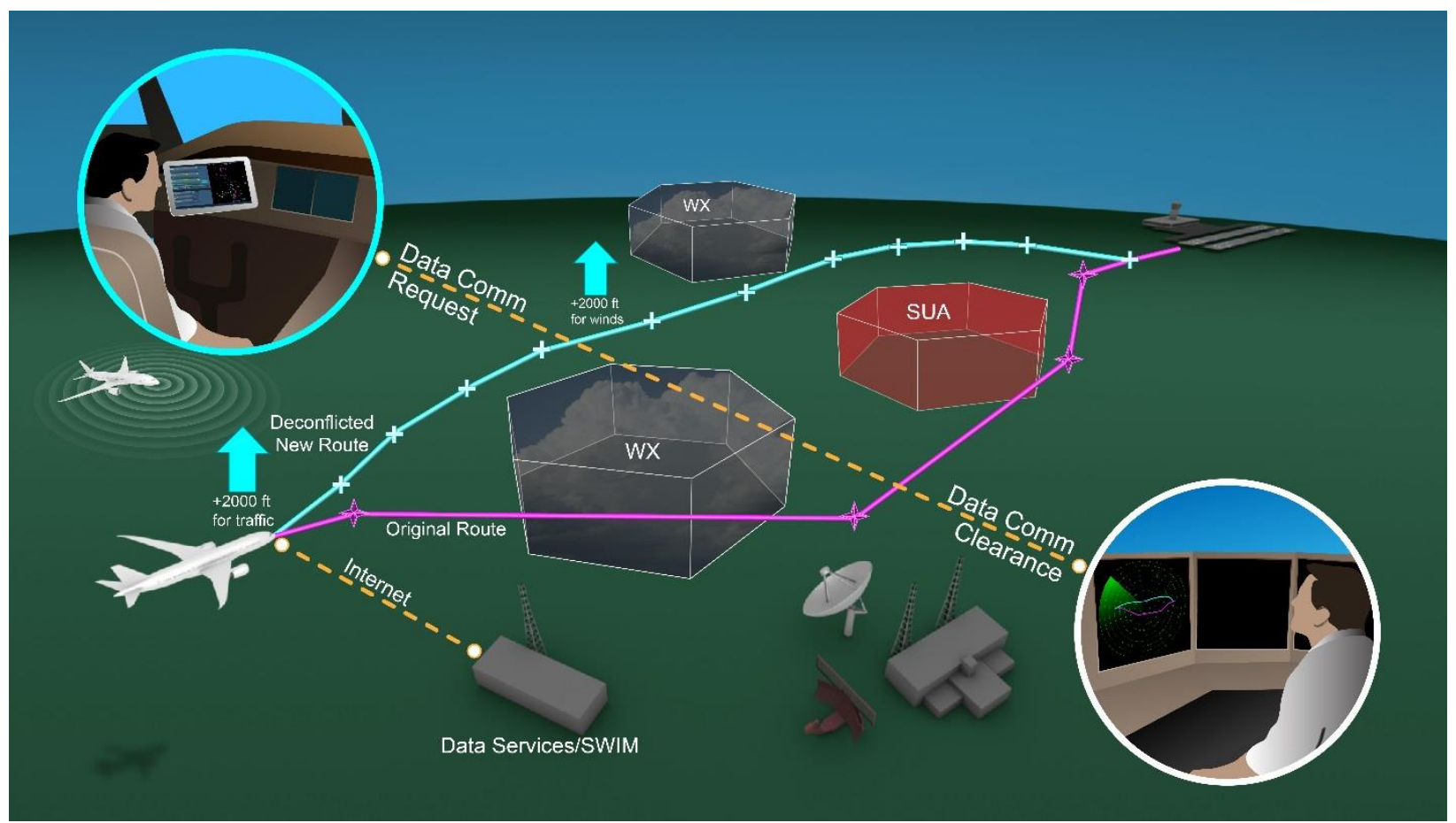

Figure 4. ABTM Roadmap Step 2 -Digital TASAR.

network enablement and information management by using increasingly higher quality and more robust external information sources. Coordination with the airlines' operational control centers is both simplified and enhanced by data communications, enabling an airline to consider dynamic network optimization of its fleet in the process of defining individual optimization objectives. This aspect of Digital TASAR may occur in ABTM 1 at some carriers, using Aircraft Communications Addressing and Reporting System (ACARS), already part of standard aircraft equipage.

Digital TASAR functionality could continue to be hosted in a similar manner as Basic TASAR: hosted in an uninstalled EFB with connectivity via an installed Aircraft Interface Device (AID) as shown in Fig. 2, hosted in an installed certified EFB, or hosted as an application in other certified avionics in the aircraft, such as the surveillance system as shown in Fig. 3. To enable implementations using certified systems, application standards and avionics architectures will need to be created. Automatic connectivity via data communications between the airplane and both ATC and flight dispatch, coupled with comprehensive airborne surveillance, improved weather and airspace status information from the ground, and evolutionary TAP optimization software improvements, will lead to a rapid buildup of experience and confidence in the technologies that enable the subsequent steps in this roadmap.

Coordination with ATC may include the controller's ground automation or may be performed directly with the controller. If ground automation is involved, change requests and approvals may be coordinated by the automation, with the controller providing final clearance approval. There is no change in the location of separation responsibility in Digital TASAR, which enables the certification and operational approval requirements to remain similar to Basic TASAR. Even though tactical separation assurance is still an ATC responsibility, Digital TASAR begins to preempt the need for most such interventions by ATC. As with the National Route Program (NRP), aircraft performing trajectory management using the capabilities of Digital TASAR should be given priority by the controller in conflict situations as extensions of their NRP route.

ABTM 3. 4D TASAR: Depicted in Fig. 5, 4D TASAR adds the speed and time element to dynamic trajectory optimization by including speed (or Cost Index-based speed profile) as well as lateral and vertical path changes in the recommended solutions. It also represents the integration of TASAR with FAA arrival flow management capabilities such as Time Based Flow Management (TBFM) automation and Advanced Interval Management (A-IM). When Required Time of Arrival (RTA) is in use at the destination airport, or A-IM is in use to merge and space aircraft for the approach, 4D TASAR will interface directly with these procedures. This seamless connectivity permits the use of 


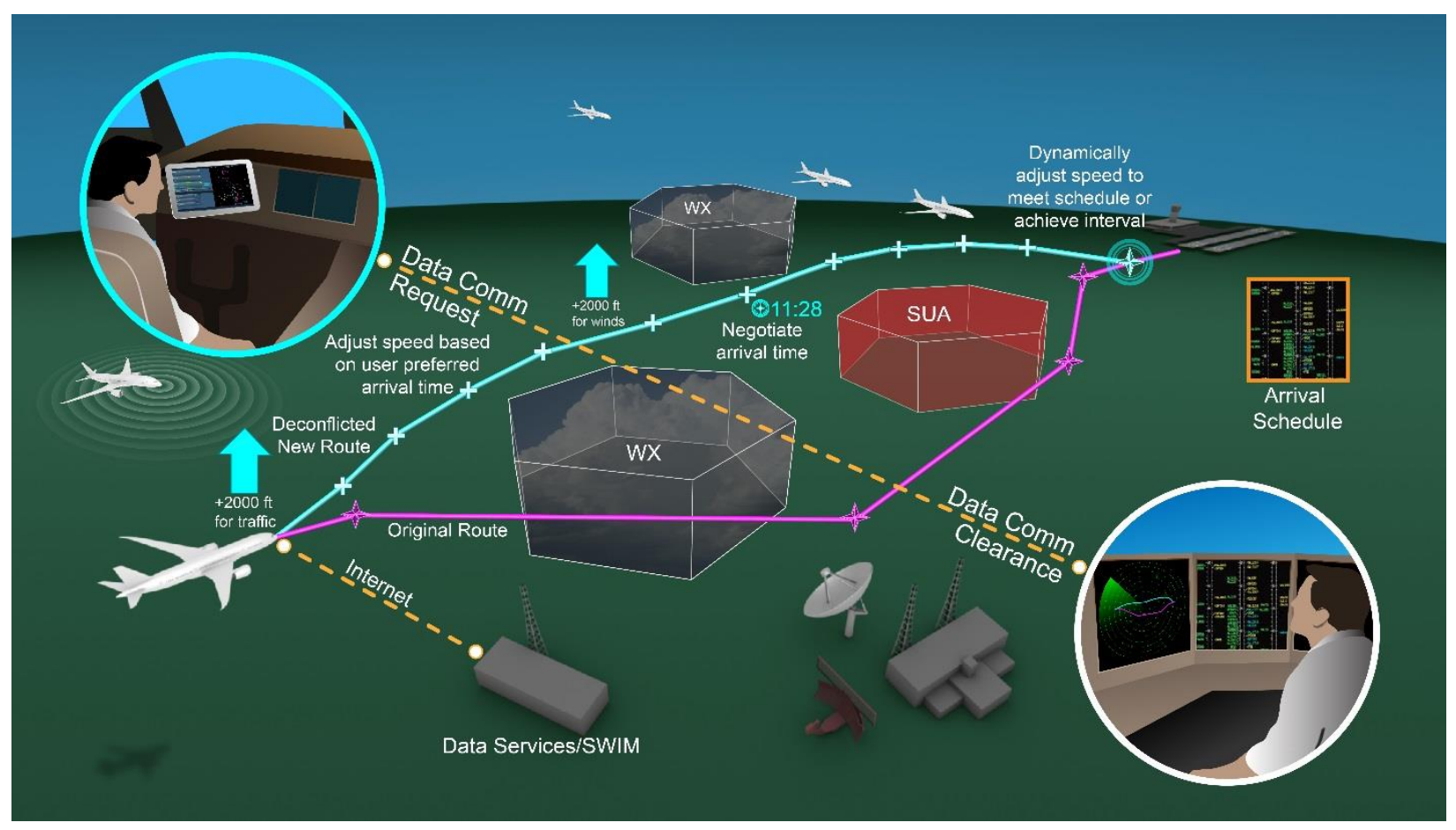

Figure 5. ABTM Roadmap Step 3 - 4D TASAR.

airborne optimization throughout the flight from departure flow to arrival flow. At this step, 4D TASAR is less likely to be implemented as an EFB application, as it more closely integrates with certified avionics that are required for RTA and A-IM.

In the cockpit, the activation of a single optimization flight guidance function after takeoff will provide lateral, vertical, and speed guidance on the flight director, with autopilot and auto throttle coupling available. Any changes in RTA would be loaded into the Flight Management System (FMS) and be accessible by TAP. When approaching a terminal area employing Advanced Interval Management, A-IM instructions will be received via Data Comm and will be executed by the A-IM system or FMS depending on aircraft specific implementation. TAP-generated trajectory modifications will take the A-IM clearances into account in creating and filtering solutions. Thus the 4D TASAR application serves as an integrated trajectory management function, effectively integrating the time-based and speedbased requirements of TBFM and IM, respectively, into the single trajectory optimization of the own ship aircraft.

TAP-recommended trajectory management solutions will thus be TBFM and/or A-IM compliant and will appear on the navigation display and on the Communications Management Unit (CMU) interface for request and response from the ATC system. When a desired solution appears, touching the "Request from ATC" button on the CMU sends the request as a data message to ATC. ATC evaluation of these requests may still be accomplished manually by the controller but ideally will be an automatic function within the FAA's En Route Automation Modernization (ERAM) system. When the trajectory amendment is approved, it is returned by a Data Comm message from ATC, appearing graphically on the Navigation Display, in a "pending" color along with the active route, and in the FMS. The FMS Execute button makes it the new active route and erases the old one. Speed and vertical portions of the new trajectory are shown alpha-numerically on the navigation display and as guidance on the flight director. The new trajectory can also be flown through the autopilot and auto throttles as in other navigation modes. Separation responsibility still remains a ground function in 4D TASAR, but the use of TAP's de-confliction feature makes intervention by ATC a rare event.

4D TASAR integrated with A-IM may not be easy to host on an EFB because of the extensive connectivity to the A-IM applications. It is likely that the automation systems for 4D TASAR and the later roadmap steps will be hosted in certified avionics containing other ADS-B IN applications. This architecture, shown in Fig. 3, is the same as the integrated option for Digital TASAR with the necessary addition of A-IM and other ADS-B IN applications in the surveillance unit or other appropriate avionics. On those aircraft also implementing A-IM, the inputs, outputs, displays, 
and peripherals are the same as for A-IM alone, so no additional hardware upgrades are required solely for the implementation of 4D TASAR.

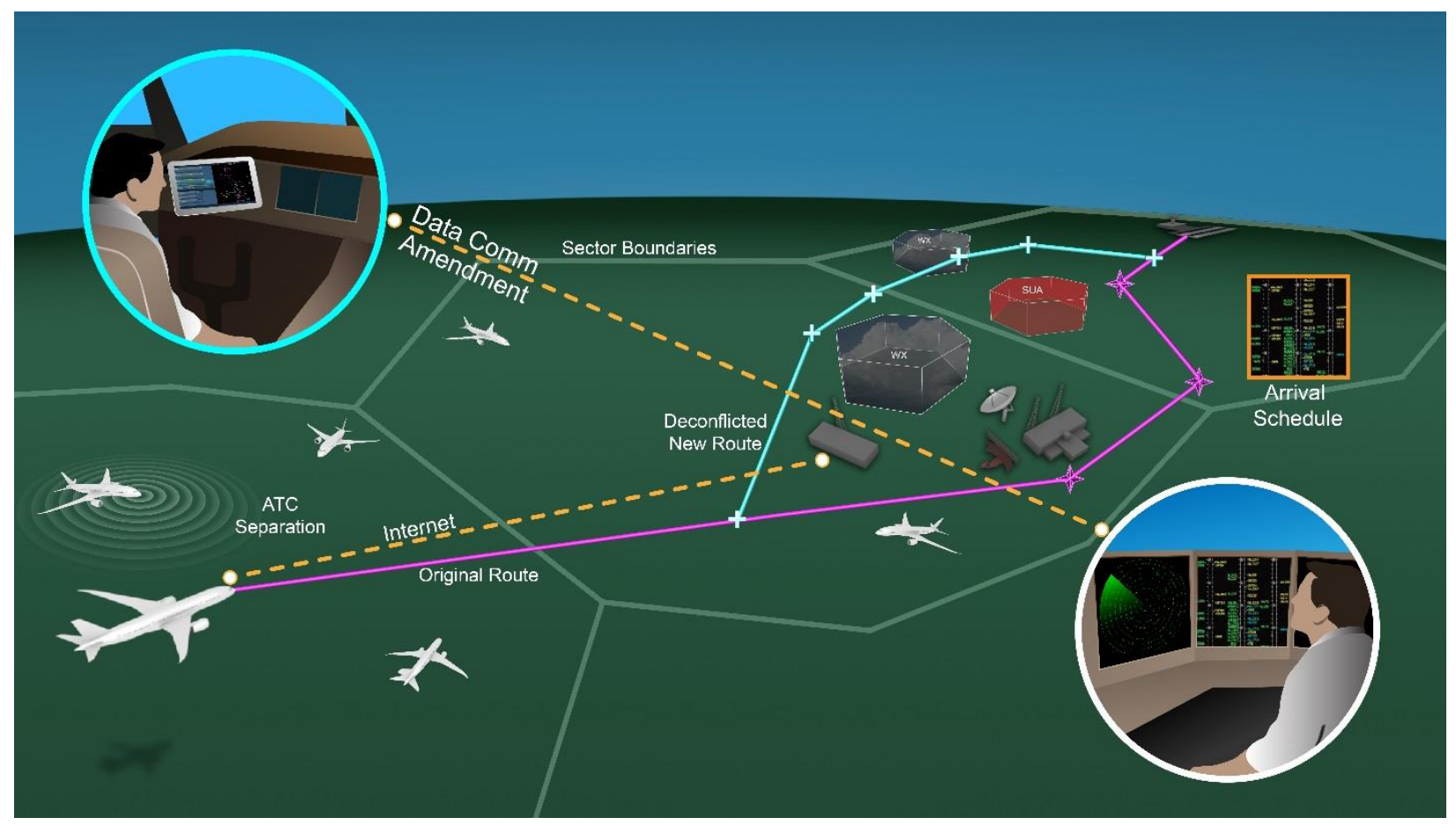

Figure 6. ABTM Roadmap Step 4 - Strategic ABTM.

The hardware upgrades required for transitioning to 4D TASAR and integration with A-IM can also accomplish the TAP application connections to the FMS and primary flight displays. The availability of TASAR benefits to the first adopter may simplify the investment case for getting both Data Comm and A-IM on the aircraft as the latter's benefits are dependent on the ground system automation being in place in order to use the function.

ABTM 4. Strategic ABTM: Depicted in Fig. 6, this step builds upon the operational experience and technology maturity gained in previous roadmap steps (Basic TASAR, Digital TASAR, and 4D TASAR), and it begins to use the separation management capability already built into these earlier technologies. In Strategic ABTM, route changes generated by the airborne system (for re-optimizing the business trajectory) that begin in the next ATC sector beyond the one presently occupied are routinely (and eventually automatically) accepted with a blanket approval by ATC automation on the basis of their de-confliction in that downstream sector. This is similar to how controllers ensure aircraft are initially deconflicted before handoff.

TAP solutions beginning in the next sector will be reviewed and approved by the flight crew for their calculated time and fuel outcomes and their "reasonableness" from a flying standpoint, as a final check on acceptability before sending the trajectory change securely to the ATC system, via Data Comm, to update the cleared trajectory of the aircraft from the identified downstream point. This trajectory message will be flagged to the Center computer showing that it has been de-conflicted by TAP using Strategic ABTM, that it conforms to the ATC airspace structure, and it therefore does not require additional controller review and approval before replacing the active clearance. Initially, traffic managers will review the Strategic ABTM changes and will have plenty of time to message the aircraft if there's an issue with the change due to constraints unknown to the airborne system. Over time, as the airborne system becomes more aware of these constraints, the need for their intervention is expected to dissipate.

Recognition of the trajectory change flag is the only modification to the ground automation required by Strategic ABTM. To the downstream controller, it will appear the same as if the route had been modified by the controller in the sector currently containing the aircraft. But because it begins in the next sector, it is of no interest to the controller in the active sector. This dramatically reduces the workload of both the current sector controller and the downstream controller because they no longer have to take any action on these trajectory modifications. 




Figure 7. ABTM Roadmap Step 5 - Full ABTM.

Once Strategic ABTM is in place, the TAP logic will establish the forward part of the trajectory modification first, as it doesn't require approval from the ground. Then a Digital TASAR request to the current sector controller will be issued, usually a "direct" to the first modified waypoint in the next sector, which will be deconflicted and therefore generally approved. ABTM 4 does not change the locus of responsibility for separation; that remains with the current sector controller. The fact that the trajectory of a given flight has changed in a downstream sector is not problematic because the change occurs before the aircraft is handed off to the receiving sector. The receiving controller will not have been concerned about that trajectory before the handoff takes place and when he does accept the handoff, the trajectory will have already been strategically de-conflicted by the TAP software. Thus no change is required in separation responsibility and as a result, certification of the TAP software is simplified.

Just as the flight plans accepted in the National Route Program today have been optimized during pre-flight planning and are treated by controllers as "the airplane not to move" in a conflict scenario, the optimized trajectories delivered to the ATC system by Strategic ABTM, because they are initially de-conflicted, will be afforded the same consideration, further improving the value of this operation to the participants. Such trajectory modifications generated by onboard automation, once sent to the ground, become the "cleared" route by ATC, and therefore the active route in the FMS. The possibility of increased workload for pilots or controllers under certain failure scenarios likely sets the FEC of Strategic ABTM at "Minor."11 While responsibility for separation remains with ATC in this roadmap step, ATC intervention with these flights will be rare, and experience with airborne separation functions will be accumulated and will help to validate the self-separation functionality required for the next roadmap step, Full ABTM.

ABTM 5. Full ABTM: As depicted in Fig. 7, in Full ABTM, tactical separation functionality has been enabled in the aircraft system, permitting equipped, operationally approved flights to operate with significant autonomy in mixed airspace, i.e., airspace shared with traditionally managed flights. With the addition of tactical separation functionality to the onboard automation, the distinction between Strategic ABTM and Full ABTM is that the aircraft's route change may begin at any time (once it has been deconflicted) and not just in downstream sectors. Along with this authority to dynamically replan and execute comes the responsibility for detecting and resolving traffic conflicts along the FMS active route. The enabling automation must therefore integrate the strategic and tactical functions of trajectory and separation management. NASA has prototyped and extensively tested such an integrated tool, the Autonomous Operations Planner (AOP). ${ }^{6}$ 
Performing the separation function on board an IFR flight currently takes place only when using visual procedures and when delegated to the pilot by the controller. The NextGen plans include automation-assisted airborne separation in both delegated and general operations but this represents a significant change in responsibility that will require careful scrutiny. Over the years in which the AOP-derived separation algorithms are exercised in TAP's de-confliction function, recorded data can be used for further analysis in support of the safety case for its use in the Full ABTM separation function. From 2020 onward, ubiquitous ADS-B OUT coverage plus Traffic Information Service Broadcast (TIS-B) and Traffic Alert and Collision Avoidance System (TCAS) / transponder-based surveillance will provide fulltime cooperative surveillance of all surrounding traffic, a requirement of Full ABTM. Through safety case analyses, issues of surveillance range, reliability, missed alerts, and false alarms can be evaluated and verified against safety performance requirements of the stand-alone separation function in Full ABTM. Once proven, this capability will form the basis of a new set of flight rules - Autonomous Flight Rules (AFR) - offering unprecedented flexibility in the choice of flight trajectory in non-VFR environments. As is the case for VFR and Instrument Flight Rules (IFR), this new set of operating rules will govern responsibilities, required equipment, training, and procedures. Since AFR will not be mandated, it will be designed to co-exist with IFR. The position and planned trajectories of AFR flights will be transmitted into the ATC system, and these flights will be included in destination TBFM. With separation responsibility being onboard and not with controllers, AFR flights will be exempt from IFR constraints that are derived from the controller's separation responsibility in en-route airspace including departure fix constraints, airspace Ground Delay Programs (GDP), Miles-In-Trail (MIT) restrictions, sector capacity limits, and ATC vectoring and speed control instructions. Removing these constraints provides more than sufficient operational benefit to Full ABTM early adopters to justify the adoption costs.

It is noted, due to the special nature of small UAS operating at low altitudes in much greater numbers than conventional IFR flights today, it is expected that AFR-like operations may be implemented initially in those operations as they can be performed in airspace where no ground-based surveillance or communications exist. It can also be achieved without pilot or controller involvement, completely automatically and safely among the vehicles themselves.

Full ABTM (ABTM 5) does not require any additional avionics hardware beyond what was described for the certified, connected version of Digital TASAR (ABTM 2). However, the software in Full ABTM now performs the additional separation function that carries a higher safety requirement. It is likely that dual TAP equipment will be needed to meet the safety requirement which will likely have FEC of "Major." ${ }^{11}$ With TCAS still contributing to the collision safety function and Full ABTM responsible for preventing the loss of standard separation, the certification level may not change, but this will have to be determined by a detailed safety analysis. The approved certification level will set the requirement for the Full ABTM architecture as determined through this further analysis. Information connectivity within the airplane may be somewhat expanded with the likely addition of synthetic-voice tacticalseparation commands (e.g., "Turn right 20 degrees for traffic" similar to ATC separation instructions), the direct input of the ABTM-cleared trajectory into the FMS, and coupling with the autopilot and flight director.

\section{The Roadmap Motivation}

A primary motivation for creating a roadmap to increase levels of user autonomy, is to take advantage of the United States' vast, underused resource, its airspace, to permit unfettered access and previously unattainable flexibility in the use of aircraft of all types for a myriad of purposes. Safety in flight operations is maintained today through the excellent performance of the FAA's ATC system and the skilled professionals who operate it. This system has grown over the past nearly ninety years from modest beginnings to one of the most complex assemblages of machines, automation, people, and procedures ever created. Over a period of extensive growth in air-traffic demand, the air traffic services have achieved and maintained an exemplary safety record, but the paradigm for how air traffic control is conducted has been essentially unchanged for more than 50 years. Our legacy system of manual control requires regimentation of the airspace and traffic flows so that human controllers have the ability to maintain order among all the aircraft in each sector so as to prevent a loss of separation between any of them. The rigidity of the airspace structure and flight procedures to accommodate IFR flights in this way have also created constraints that impose delay and inefficiency on air traffic as a necessary price of admission to the airspace. Procedures such as departure fix metering, "playbook" weather re-routes, GDP, MIT restrictions, speed restrictions, and sector capacity restrictions all represent the constraints on operational flexibility for airspace users that can be overcome through the systematic increases in operational autonomy afforded by following this roadmap. These steps make it possible to achieve the airspace user's goal of broad operational flexibility while maintaining active participation in, and conformance with, the centralized traffic flow management exercised on and around an airport (departure and arrival). 
The FAA's NextGen program is designed to usher in new technologies for communication, navigation, and surveillance applied to air navigation and air traffic control. For the airspace user, these technologies represent a major investment commitment that requires justification through operational savings. The motivation for the ABTM Roadmap proposed here is to build the airspace user's business case for flight in NextGen through a series of economically and technically attainable steps to remove the constraints to flight and permit the use of dynamically optimized flight trajectories in a mixed environment of user capabilities.

\section{Air Navigation Service Provider Benefit}

While the ABTM Roadmap is "user centric" in that flight operations center on optimizing the aircraft operator's desired trajectory from origin to destination, those individual operators are not the only beneficiaries of the transition. Each step in the roadmap also provides a benefit to the Air Navigation Service Provider (ANSP) and to the efficient functioning of the National Airspace System itself. In ABTM 1, Basic TASAR enables the users to make better informed, more approvable requests for route changes than is typically achieved without the TASAR airborne technology. This makes better use of the airspace by increasing the efficiency of the flight while reducing the number of nuisance request by pilots to controllers. In ABTM 2, Digital TASAR employs the Data Comm system and connectivity to even more external data sources (such as the FAA's System Wide Information Management, or SWIM) to further enhance the user benefits and ATC approvability of change requests while reducing both pilot and controller workloads in performing the process. Voice exchanges on the frequency are significantly reduced, and no manual data entry is required by either the pilot or controller in accomplishing the more valuable trajectory amendment. In ABTM 3, 4D TASAR promotes users becoming willing and proactive participants in the time-based arrival management procedures, reducing controller workload in managing departure divergence and arrival flow "preconditioning" (i.e. vectoring and speed adjustments to establish the desired flow). The use of the speed and time dimension in the optimization solution achieves even greater efficiencies while enhancing performance of the destination airport flow management processes. The ANSP benefits continue in ABTM 4, Strategic ABTM, by reducing the burden of intersector coordination of flight plan changes. This step also takes advantage of data communications from the aircraft linking to the FAA's Traffic Flow Management System (TFMS) and ERAM software to leverage their ability to automatically evaluate the acceptability of a new proposed trajectory. Finally, in ABTM 5, Full ABTM, each flight operating under AFR reduces the load on the ATC system, effectively increasing NAS capacity without increasing system costs and enabling the ANSP to provide better services to those users for which traditional IFR operations are sufficient and desired in meeting their business objectives.

\section{The Way Forward}

The activities required to proceed with this roadmap are varied, numerous, and involve both ongoing and planned NASA-conducted research, industry coordination, and cooperation with FAA and other NASA projects. The inservice trials of Basic TASAR at Virgin America and Alaska Airlines are slated to begin in 2017. ${ }^{9}$ This activity will provide a wealth of data on actual operations that will be analyzed for the achievement of benefits and applied to improvements in the system.

The preliminary safety analysis for each roadmap step completed early this year ${ }^{11}$ must be followed up with a more rigorous analysis of the hazards and detailed safety analyses for each roadmap step, such as was accomplished for Basic TASAR. ${ }^{10}$ NASA can prototype the new capabilities representing advanced ABTM steps through flight test and partnering efforts to assist the industry in assessing their value in their operations. It will primarily then become an industry driven development and implementation effort going forward, based on the expected and demonstrated benefits. For example, cockpit avionics architectures incorporating advanced forms of the TAP software will evolve because of existing and planned avionics upgrades by various user classes, factoring in the extent to which each user intends to progress along the roadmap steps. Certification and operational approvals will be sought by the early adopter applicants. ${ }^{12}$

Human factors assessments of the human-machine interface and procedures must be performed for each succeeding step in the roadmap, as they were already conducted for Basic TASAR. ${ }^{9}$ Much of this can be done in simulation, but prototyping of equipment and flight validation of procedures will also assist in achieving the necessary approvals as we advance through these steps on the ABTM roadmap. For the last two steps in this process, additional standards work will need to be undertaken by RTCA. The government and industry together, through RTCA, must clearly define what aspects of the applications in the ABTM roadmap are already covered by standards and what will need to be standardized to ensure safety and interoperability. 


\section{Conclusion}

NASA has undertaken a road-mapping effort for Airborne Trajectory Management, founded on years of operational concept exploration, to identify the most probable path to airspace users achieving significant operational benefits, through incrementally increasing operational autonomy, by enabling aircraft to dynamically adjust their business trajectories in flight according to their mission needs. The result of this road-mapping effort is five principal steps building from Basic TASAR in-flight optimization and incrementally providing increasing levels of capability while ensuring first adopter benefits. Each step serves as a technology validation for the next step, facilitating certification and operational approval and smooth integration into the NAS. The existing NextGen programs for Data Communications, Time Based Flow Management and terminal area Interval Management are leveraged by this roadmap as enablers for real operating benefits arising from Airborne Trajectory Management. The roadmap steps are designed for evolutionary implementation while minimizing repetitious investments by users in hardware and certification. Each roadmap step is supportable on its own merits, enabling the choice by each operator on how far to proceed on the roadmap. All roadmap steps support mixed operations and per-aircraft implementation, such that early adopters may achieve early benefits on even the first-equipped flights. Benefits of Airborne Trajectory Management to the Air Navigation Service Provider are also described. The initial steps of the roadmap are already in progress through NASA partnerships with airlines. The intermediate steps align well with FAA NextGen programs and will help users justify equipage for the programs. Accomplishing later steps will follow, depending on the expressed needs of the user community.

\section{References}

1. Ballin, M., Wing, D., "Traffic-Aware Strategic Aircrew Requests (TASAR)", 12th AIAA Aviation Technology, Integration, and Operations (ATIO) Conference, AIAA-2012-5623, 2012.

2. Henderson, J., "Traffic Aware Strategic Aircrew Requests (TASAR) Concept of Operations", NASA Contractor Report, NASA/CR-2013-218001, 2013.

3. Wing, D. and Cotton, W.B., "For Spacious Skies: Self-Separation with 'Autonomous Flight Rules' in US Domestic Airspace," 11th AIAA ATIO Conference, AIAA-2011-6865, 2011.

4. Wing, D. and Cotton, W.B., "Autonomous Flight Rules: A Concept for Self-Separation in U.S. Domestic Airspace," NASA/TP-2011-217174, 2011.

5. Federal Aviation Administration, "A Report from the ADS-B IN Aviation Rulemaking Committee to the Federal Aviation Administration," September 30, 2011.

6. Karr, D.A., Vivona, R.A., Roscoe, D.A., DePascale, S.M., and Wing, D.J., "Autonomous Operations Planner: A Flexible Platform for Research in Flight-Deck Support for Airborne Self-Separation," $12^{\text {th }}$ AIAA ATIO Conference, AIAA-2012-5417, 2012.

7. Wing, D., Ballin, M., Koczo, S., Vivona, R., "Developing an On-Board Traffic Aware Flight Optimization Capability for Near-Term Low-Cost Implementation", AIAA-2013-4231, 2013.

8. Woods, S.E., Vivona, R.A., Henderson, J., Wing, D.J., and Burke, K.A., "Traffic Aware Planner for Cockpitbased Trajectory Optimization", accepted to $16^{\text {th }}$ AIAA ATIO Conference, 2016.

9. Wing, D.J., "Achieving TASAR Operational Readiness," 15 ${ }^{\text {th }}$ AIAA ATIO Conference, AIAA-2015-3400, 2015.

10. Koczo, S., "Analysis of Operational Hazards and Safety Requirements for Traffic Aware Strategic Aircrew Requests (TASAR)", NASA Contractor Report, NASA/CR-2013-218002, 2013.

11. Cotton, W., Hilb, R., Koczo, S., Wing, D., "Preliminary Assessment of Operational Hazards and Safety Requirements for Airborne Trajectory Management (ABTM) Roadmap Applications", NASA Technical Memorandum, NASA/TM-2016-219176, 2016.

12. Koczo, S., "TASAR Certification and Operational Approval Requirements - Analyses and Results", NASA Contractor Report, NASA/CR-2015-218708, 2015. 